\title{
QED Mesons, the QED Neutron, and the Dark Matter
}

\author{
Cheuk-Yin Wong ${ }^{1 *}$ \\ ${ }^{1}$ Physics Division, Oak Ridge National Laboratory", Oak Ridge, Tennessee 37831, USA
}

\begin{abstract}
Schwinger's boson solution for massless fermions in QED in 1+1D has been applied and generalized to quarks interacting in QED and QCD interactions, leading to stable and confined open-string QED and QCD boson excitations of the quark-QCD-QED system in 1+1D. Just as the open-string QCD excitations in 1+1D can be the idealization of QCD mesons with a flux tube in 3+1D, so the open-string QED excitations in 1+1D may likewise be the idealization of QED mesons with masses in the tens of $\mathrm{MeV}$ region, corresponding possibly to the anomalous X17 and E38 particles observed recently. A further search for bound states of quarks interacting in the QED interaction alone leads to the examination on the stability of the QED neutron, consisting of two $d$ quarks and one $u$ quark. Theoretically, the QED neutron has been found to be stable and estimated to have a mass of $44.5 \mathrm{MeV}$, whereas the analogous QED proton is unstable, leading to a long-lived QED neutron that may be a good candidate for the dark matter.
\end{abstract}

\section{Introduction}

The quark-QCD-QED vacuum is the lowest-energy state of the quark-QCD-QED system with quarks filling up the (hidden) negative-energy Dirac sea and interacting with the QCD (quantum chromodynamical) interaction and the QED (quantum electrodynamical) interaction. It is defined as the state with no valence quark above the Dirac sea and no valence antiquark as a hole below the Dirac sea. A local disturbance will generate stable collective excitations of the quark-QCD-QED system as in a fluid. There are stable and confined collective QCD excitations showing up as QCD mesons, such as $\pi, \eta$, and $\eta^{\prime}$. The question of our central interest is whether there may also exist stable QED excitations of the quark-QCD-QED system showing up as QED mesons in the mass region of many tens of $\mathrm{MeV}$, together with the related QED neutron with $d-u-d$ quarks stabilized by the QED interaction.

Such a question may appear preposterous as one can surmise from the following debate between a Wise Guy and an Explorer. The Wise Guy objects and argues that quarks interact in QCD and QED simultaneously. When quarks and antiquarks interact, their color charges and electric charges interact simultaneously. A stable QED excitation of the quarks cannot be independent and cannot occur without the simultaneous QCD excitation. To these

\footnotetext{
*Email:wongc@ornl.gov

** This manuscript has been authored in part by UT-Battelle, LLC, under contract DE-AC05-00OR22725 with the US Department of Energy (DOE). The US government retains and the publisher, by accepting the article for publication, acknowledges that the US government retains a nonexclusive, paid-up, irrevocable, worldwide license to publish or reproduce the published form of this manuscript, or allow others to do so, for US government purposes. DOE will provide public access to these results of federally sponsored research in accordance with the DOE Public Access Plan (http://energy.gov/downloads/doe-public-access-plan), Oak Ridge, Tennessee 37831, USA
}

objections, the Explorer defends and replies that the quark currents, as well as the QCD and the QED gauge fields, are not single-element quantities. They are $3 \times 3$ color matrices with 9 matrix elements that separate naturally into $\mathbf{3} \otimes \mathbf{3}^{*}=\mathbf{1} \oplus \mathbf{8}$, with a color-singlet subgroup $\mathbf{1}$ and a coloroctet subgroup $\mathbf{8}$ executing collective dynamics separately. In the space-time arena, there can be localized QCD excitations of the color-octet current leading to QCD mesons. There can also be independent QED excitations of the color-singlet current showing up as QED mesons at different energies. The Wise Guy objects further and says that only the non-Abelian QCD interaction can confine quarks, and the QED interaction does not confine particles such as electrons and positrons. Thereupon, the Explorer explains that light quarks can be considered approximately massless, and the application of the Schwinger's solution $[1,2]$ indicates that massless quarks in QED are confined in $1+1 \mathrm{D}$. The confined open string in $1+1 \mathrm{D}$ can be considered to be the idealization of a flux tube in $3+1 \mathrm{D}$. Gribov showed that massless fermions in QED in 3+1D are confined [3, 4]. Furthermore, lattice gauge calculations show that fermions in QED in 3+1D can be confined under appropriate conditions $[5,6]$. Clearly, whatever the outcome of the debate may be, it is necessary to confront the predictions on the QED mesons and the QED neutron of the quark-QCD-QED system with experiments. The present investigation facilitates such a confrontation.

\section{Generalization of Schwinger's solution from QED to (QED+QCD) in 1+1D}

We first summarize the dynamics of the Schwinger's QED solution for massless fermions in 1+1D as reviewed in [7]. Subject to a QED gauge field disturbance $A^{\mu}$ with a coupling constant $g_{2 \mathrm{D}}$ in $1+1 \mathrm{D}$, the massless fermion field $\psi(x)$ 
satisfies the Dirac equation,

$$
\gamma_{\mu}\left(p^{\mu}-g_{2 \mathrm{D}} A^{\mu}\right) \psi=0 .
$$

The disturbance $A^{\mu}$ instructs the fermion field $\psi$ how to move, and through the Maxwell equation,

$$
\partial_{\mu} F^{\mu v}=\partial_{\mu}\left(\partial^{\mu} A^{v}-\partial^{v} A^{\mu}\right)=g_{2 \mathrm{D}} j^{v}=g_{2 \mathrm{D}} \bar{\psi} \gamma^{v} \psi,
$$

the fermion field $\psi$ in turn generates the current $j^{\mu}$ which instructs the gauge field $A^{\mu}$ how to act. A stable collective excitation of the fermion-QED system occurs, when the disturbance $A^{\mu}$ gives rise to the current $j^{\mu}$ which in turn leads to the gauge field $A^{\mu}$ self-consistently. By imposing the Schwinger modification factor to ensure the gauge invariance of the fermion Green's function, the fermion current $j^{\mu}(x)$ at the space-time point $x$ induced by $A^{\mu}$ can be evaluated. After the singularities from the left and from the right cancel, the fermion current $j^{\mu}$ is found to relate explicitly to the perturbing QED gauge field $A^{\mu}$ by $[1,2]$

$$
j^{\mu}=-\frac{g_{2 \mathrm{D}}^{2}}{\pi}\left(A^{\mu}-\partial^{\mu} \frac{1}{\partial_{\lambda} \partial^{\lambda}} \partial_{v} A^{v}\right) .
$$

Upon substituting such a relation to the Maxwell equation (2), we get $j^{\mu}$ and $A^{\mu}$ both satisfy the Klein-Gordon equation

$$
\partial_{\nu} \partial^{\nu} A^{\mu}+\frac{g_{2 \mathrm{D}}^{2}}{\pi} A^{\mu}=0, \text { and } \partial_{\nu} \partial^{\nu} j^{\mu}+\frac{g_{2 \mathrm{D}}^{2}}{\pi} j^{\mu}=0,
$$

for a boson with a mass given by $m=g_{2 \mathrm{D}} / \sqrt{\pi}$.

The Schwinger model can be generalized from QED to (QED+QCD) $[8,9]$. Because of the three-color nature of quarks, the quark currents and the QED and QCD gauge fields are $3 \times 3$ color matrices which can be expanded in terms of the nine generators of the $\mathrm{U}(3)$ group,

$$
j^{\mu}=\sum_{i=0}^{8} j_{i}^{\mu} t^{i}, \quad A^{\mu}=\sum_{i=0}^{8} A_{i}^{\mu} t^{i}, \quad t^{0}=\frac{1}{\sqrt{6}}\left(\begin{array}{lll}
1 & 0 & 0 \\
0 & 1 & 0 \\
0 & 0 & 1
\end{array}\right),
$$

where $t^{0}$ is the generator of the U(1) color-singlet subgroup and $t^{1}, t^{2}, \ldots, t^{8}$ are the eight generators of the $\mathrm{SU}(3)$ color-octet subgroup. Because the current and the gauge field of each subgroup depend on each other within the subgroup, but do not depend on the current and the gauge field of the other subgroup, the two different currents and the gauge fields in their respective subgroups possess independent stable QED and QCD collective excitations at different energies. For the QCD interaction, stable QCD collective excitations can be attained in restricted variations which keep the orientation of the unit vector $\tau^{1}$ in the color-octet generator space fixed while their amplitudes $A_{1}^{\mu}$ are allowed to vary. That is, with

$$
j^{\mu}=j_{0}^{\mu} \tau^{0}+j_{1}^{\mu} \tau^{1}, \quad A^{\mu}=A_{0}^{\mu} \tau^{0}+A_{1}^{\mu} \tau^{1},
$$

where $\tau^{0}=t^{0}, \tau^{1}=\sum_{i=1}^{8} n_{i} t^{i}$, with fixed direction cosines $n_{i}=2 \operatorname{tr}\left\{\tau^{1} t^{i}\right\}$ in the color-octet generator space. With the above currents and gauge fields (6) in the color-singlet and the color-octet subgroups, Schwinger's solution for QED can be trivially generalized to (QED+QCD) leading to the currents and gauge fields satisfying their corresponding
Klein-Gorden equations with masses depending on their respective coupling constants $[8,9]$.

Consequently, there are independent collective QED and QCD excitations of the quark-QCD-QED system in $1+1 \mathrm{D}$ where these excitations can be described as openstring states of $q \bar{q}$ pairs. We note perceptively that in $1+1 \mathrm{D}$, the open string does not have a structure, but the coupling constant $g_{2 \mathrm{D}}$ has the dimension of a mass. In contrast in $3+1 \mathrm{D}$, the flux tube has a structure with a radius $R_{T}$, but the coupling constant $g_{4 \mathrm{D}}$ is dimensionless. The $1+1 \mathrm{D}$ open string can be considered an idealization of the physical meson in $3+1 \mathrm{D}$ with a flux tube of radius $R_{T}$, if the coupling constants $g_{2 \mathrm{D}}$ and $g_{4 \mathrm{D}}$ in the two different space-time dimensions are related by $[8,9]$

$$
g_{2 \mathrm{D}}^{2}=g_{4 \mathrm{D}}^{2} /\left(\pi R_{T}^{2}\right)=4 \alpha / R_{T}^{2} .
$$

Consequently, the masses of the QED and QCD mesons in $3+1 \mathrm{D}$ in the open-string description are approximately

$$
m_{\mathrm{QCD}}^{2}=4 \alpha_{\mathrm{QCD}} / \pi R_{T}^{2}, \quad m_{\mathrm{QED}}^{2}=4 \alpha_{\mathrm{QED}} / \pi R_{T}^{2} .
$$

\section{QCD and QED mesons as open strings}

To get a better determination of the QCD and QED meson masses, it is necessary to take into account the flavor mixtures $D_{i j}$ and the quark rest masses $m_{f}$. Using the method of bosonization, we obtain the semi-empirical mass formula for the neutral QCD mesons with $N_{f}=3$ [9]

$$
m_{i}^{2}=\left(\sum_{f=1}^{N_{f}} D_{i f}\right)^{2} \frac{4 \alpha_{\mathrm{QCD}}}{\pi R_{T}^{2}}+m_{\pi}^{2} \sum_{f=1}^{N_{f}} \frac{m_{f}}{\left(m_{u}+m_{d}\right) / 2}\left(D_{i f}\right)^{2},
$$

and for QED with $N_{f}=2$ and isospin $\left(I, I_{3}=0\right)$,

$$
m_{I}^{2}=\left[\frac{Q_{u}+(-1)^{I} Q_{d}}{\sqrt{2}}\right]^{2} \frac{4 \alpha_{\mathrm{QED}}}{\pi R_{T}^{2}}+m_{\pi}^{2} \frac{\alpha_{\mathrm{QED}}}{\alpha_{\mathrm{QCD}}} .
$$

where $Q_{u}=2 / 3$ and $Q_{d}=-1 / 3$ are the charge numbers of $u$ and $d$ quarks respectively. With the flavor mixture and quark masses as given by the Particle Data Group [10], $\alpha_{\mathrm{QED}}=1 / 137, \alpha_{\mathrm{QCD}}=0.68 \pm 0.08$, and $R_{T}=0.40 \pm 0.04$ $\mathrm{fm}$, we obtain the theoretical QCD and QED meson masses in Table 1.

Table 1. The experimental and theoretical masses of neutral, $I_{3}=0$, QCD and QED mesons, obtained with the semi-empirical mass formula (9) for QCD mesons and (10) for QED mesons.

\begin{tabular}{|c|c|c|c|c|}
\cline { 3 - 5 } \multicolumn{2}{c|}{} & {$\left[I\left(J^{\pi}\right)\right]$} & $\begin{array}{c}\text { Experimental } \\
\text { mass } \\
(\mathrm{MeV})\end{array}$ & $\begin{array}{c}\text { Semi-empirical } \\
\text { mass } \\
\text { formula } \\
(\mathrm{MeV})\end{array}$ \\
\hline QCD & $\pi^{0}$ & {$\left[1\left(0^{-}\right)\right]$} & $134.9768 \pm 0.0005$ & $134.9^{\ddagger}$ \\
meson & $\eta$ & {$\left[0\left(0^{-}\right)\right]$} & $547.862 \pm 0.017$ & $498.4 \pm 39.8$ \\
& $\eta^{\prime}$ & {$\left[0\left(0^{-}\right)\right]$} & $957.78 \pm 0.06$ & $948.2 \pm 99.6$ \\
\hline QED & $\mathrm{X} 17$ & {$\left[0\left(0^{-}\right)\right]$} & $16.94 \pm 0.24^{\#}$ & $17.9 \pm 1.5$ \\
meson & $\mathrm{E} 38$ & {$\left[1\left(0^{-}\right)\right]$} & $37.38 \pm 0.71^{\oplus}$ & $36.4 \pm 3.8$ \\
\hline
\end{tabular}

$¥$ Calibration mass

\# A. Krasznahorkay et al., arxiv:2104.10075

${ }^{\oplus}$ K. Abraamyan et al., EPJ Web Conf 204,08004(2019)

We find from Table 1 that the open-string description of the QCD and QED mesons is a reasonable concept and 
the anomalous X17 [11] and E38 [12] observed recently may be QED mesons. The parent particles of the anomalous soft photons [13] may be QED mesons.

\section{Schwinger's QED boson as a relativistic two-body problem in 1+1 dimensions}

It is desirable to construct a phenomenological two-body model for Schwinger's QED bound state in 1+1D involving a massless fermion and an antifermion interacting in an effective two-body QED interaction $\Phi_{12}$. The interaction $\Phi_{12}$ must be calibrated to yield Schwinger's exact solution in field theory. Neglecting spins, the relativistic two-body wave equations for the wave function $\Psi$ for the QED interactions in 1+1 dimensions consist of two mass-shell constraints on each of the interacting particles $[15,16]$,

$$
\begin{aligned}
& \mathcal{H}_{1}|\Psi\rangle=\left\{p_{1}^{2}-m_{1}^{2}-\Phi_{12}\left(x_{12 \perp}\right)\right\}|\Psi\rangle=0, \\
& \mathcal{H}_{2}|\Psi\rangle=\left\{p_{2}^{2}-m_{2}^{2}-\Phi_{12}\left(x_{21 \perp}\right)\right\}|\Psi\rangle=0 .
\end{aligned}
$$

In the center-of-mass frame where the particle momentum is $p_{i}=\left(\epsilon_{i}, q_{i}\right)$, the above two-body wave equations become

$$
\begin{aligned}
& \epsilon_{1}^{2}|\Psi\rangle=\left\{q_{1}^{2}+m_{1}^{2}+\Phi_{12}\left(x_{12 \perp}\right)\right\}|\Psi\rangle=0, \\
& \epsilon_{2}^{2}|\Psi\rangle=\left\{q_{2}^{2}+m_{2}^{2}+\Phi_{12}\left(x_{12 \perp}\right)\right\}|\Psi\rangle=0,
\end{aligned}
$$

and the mass of the bound states is $m=\epsilon_{1}+\epsilon_{2}$. By requiring $\Phi_{12}$ to give the mass $m=g_{2 \mathrm{D}} / \sqrt{\pi}$ in a linearly confining potential to match Schwinger's exact solution for massless fermions in QED in 1+1D, we find [14]

$$
\Phi_{12}\left(x_{12 \perp}\right)=\frac{2 \epsilon_{1} \epsilon_{2}}{\epsilon_{1}+\epsilon_{2}}\left(-Q_{1} Q_{2}\right) \kappa\left|x_{1}-x_{2}\right|, \quad \kappa=\frac{g_{2 \mathrm{D}}^{2}}{4 \pi},
$$

where $Q_{1}$ and $Q_{2}$ are the charge numbers and $x_{1}$ and $x_{2}$ are the spatial coordinates of the interacting particles. We shall use $\Phi_{12}$ to study the stability of the QED neutron with quarks interacting in QED.

\section{The Stability of the QED Neutron}

The success of the open-string description of the QCD and QED mesons leads to the search for other neutral quark systems stabilized by the QED interaction between the constituents in the color-singlet subgroup, with the coloroctet QCD gauge interaction as a spectator field. Of particular interest is the QED neutron with the $d, u$, and $d$ quarks [14]. They form a color product group of $\mathbf{3} \otimes \mathbf{3} \otimes \mathbf{3}$ $=\mathbf{1} \oplus \mathbf{8} \oplus \mathbf{8} \oplus \mathbf{1 0}$, which contains a color singlet subgroup 1 where the color-singlet current and the color-singlet QED gauge field reside. In the color-singlet $d-u-d$ system with three different colors, the attractive QED interaction between the $d$ and $u$ quarks may overwhelm the repulsive QED interaction between the two $d$ quarks to stabilize the composite color-singlet QED neutron into a linear configuration. It is reasonable to treat the system in $1+1 \mathrm{D}$ spacetime. We generalize the two-body equations of (11a) and (11b) to the three-quark system by imposing three massshell constraints relating the momenta, the masses, and their interactions in the form

$$
\begin{aligned}
& \mathcal{H}_{1}|\Psi\rangle=\left\{p_{1}^{2}-m_{1}^{2}-\left[\Phi_{12}\left(x_{12}\right)+\Phi_{13}\left(x_{13}\right)\right]\right\}|\Psi\rangle=0, \\
& \mathcal{H}_{2}|\Psi\rangle=\left\{p_{2}^{2}-m_{2}^{2}-\left[\Phi_{21}\left(x_{21}\right)+\Phi_{23}\left(x_{23}\right)\right]\right\}|\Psi\rangle=0, \\
& \mathcal{H}_{3}|\Psi\rangle=\left\{p_{3}^{2}-m_{3}^{2}-\left[\Phi_{31}\left(x_{31}\right)+\Phi_{32}\left(x_{32}\right)\right]\right\}|\Psi\rangle=0,
\end{aligned}
$$

where $\Phi_{i j}$ is the effective two-body QED interaction (13) extracted from Schwinger's exact QED solution in 1+1D. We search for an energy minimum for the QED neutron state by using a variational wave function. It is convenient to choose a Gaussian variational wave function of the spatial dimensionless variables $y_{1}, y_{2}, y_{3}$ with standard deviations $\sigma_{1}, \sigma_{2}$, and $\sigma_{3}$ as variational parameters, for the $d$, $u$, and $d$ quarks respectively,

$$
\Psi\left(y_{1}, y_{2}, y_{3}\right)=N \exp \left\{-\frac{y_{1}^{2}}{4 \sigma_{1}^{2}}-\frac{y_{2}^{2}}{4 \sigma_{2}^{2}}-\frac{y_{3}^{2}}{4 \sigma_{3}^{2}}\right\},
$$

where $y_{i}=\sqrt{\kappa} x_{i}$. The charge numbers of the quarks are $Q_{1}=Q_{3}=-1 / 3$, and $Q_{2}=2 / 3$. For the lowest-energy state, $\sigma_{1}=\sigma_{3}$, and the variational parameters consist only of $\sigma_{1}$ and $\sigma_{2}$. We look for the state with the lowest composite mass $M=\epsilon_{1}+\epsilon_{2}+\epsilon_{3}$ in the variations of $\sigma_{1}$ and $\sigma_{2}$,

$$
\frac{\delta^{2} M\left(\sigma_{1}, \sigma_{2}\right)}{\delta \sigma_{1} \delta \sigma_{2}}=0
$$

The motion of the three quarks should maintain a fixed center of mass for the composite system. The coordinates of the three quarks must satisfy the center-of-mass condition on the spatial coordinates, $\sum_{i=1}^{3} \epsilon_{i} y_{i}=0$. The variational wave function $\Psi$ is normalized according to

$$
\int d y_{1} d y_{2} d y_{3}\left|\Psi\left(y_{1}, y_{2}, y_{3}\right)\right|^{2} \delta\left(\epsilon_{1} y_{1}+\epsilon_{2} y_{2}+\epsilon_{3} y_{3}\right)=1 \text {. }
$$

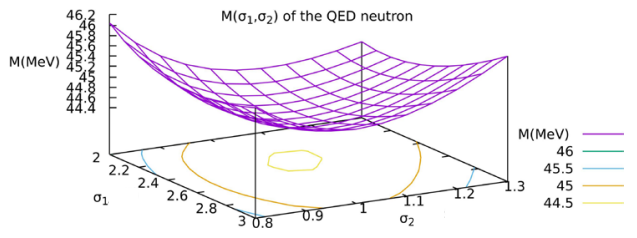

Figure 1. The mass $M$ of the QED neutron as a function of the variation parameters $\sigma_{1}$, and $\sigma_{2}$. The QED neutron has an energy minimum at $M=44.5 \mathrm{MeV}$.

By the variational calculations, we find that the QED neutron is stable [14]. Its mass $M$ has an energy minimum, $M=44.5 \mathrm{MeV}$, at $\sigma_{1}=2.40$ and $\sigma_{2}=1.05$ as shown in Fig. 1 , corresponding to $\sigma_{1} / \sqrt{\kappa}=19.9 \mathrm{fm}$ and $\sigma_{2} / \sqrt{\kappa}=8.71 \mathrm{fm}$.

We study next the stability of the QED color-singlet proton with two $u$ quarks and a $d$ quark by carrying out variational calculations similar to those for the QED neutron. The variation over a very large range of $\sigma_{1}$ and $\sigma_{2}$ fails to find an energy minimum. Extending the range of $\sigma$ will only drive the total energy of the system lower with the $u$ quarks farther and farther apart without the energy turning to a minimum. An examination of the potential 
energies of the interaction indicates that the QED proton is unstable because of the stronger repulsion between the two $u$ quarks in comparison with the weaker attractive interactions between the $d$ and the $u$ quarks. The QED proton also does not possess a continuum state with isolated quarks because the isolation of quarks is forbidden. Therefore, the QED proton does not exist as a stable bound state or a continuum state.

\section{Stability of QED Neutron against Weak Decay and Dark matter}

The absence of a QED proton state has an important consequence for the weak decay of the QED neutron, which could occur when a $d$ quark decays into a $u$ quark. Such a QED neutron weak decay would result in a possible QED proton final state, if a QED proton state could exist. Because there is no final bound or continuum QED proton state for the QED neutron to decay onto, the density of final states for the weak decay is zero. Consequently the rate for the QED neutron to decay into a QED proton is zero. The QED neutron can only decay by a baryon-number non-conserving transition which presumably has a very long life time. Therefore, the lowest energy QED neutron is a stable particle with a very long lifetime and is in fact a dark neutron. Because of its long lifetimes, the QED dark neutron may be good candidate for a part of the dark matter. Self-gravitating assemblies of QED dark neutrons may be produced by coalescence during the deconfinement-toconfinement phase transition of the quark gluon plasma in the evolution of the early Universe.

In other astrophysical frontiers, the merging of two neutron stars may lead to the production of a quark matter with deconfined quarks [17]. The coalescence of deconfined quarks during the deconfinement-to-confinement phase transition will produce QED neutrons in the postmerger environment. It has also been suggested that deconfined quark matter may be present in the core of a massive neutron star [18]. In such a neutron star, the transition region close to the core may contain QED neutron matter arising from the coalescence of deconfined quarks.

\section{Conclusion and Discussions}

In a system of quarks interacting in QCD and QED interactions, the current and the gauge fields reside in colorsinglet QED and color-octet QCD subspaces and execute independent collective excitations. The collective excitations in the color-octet sector give rise to the QCD mesons such as $\pi, \eta$, and $\eta^{\prime}$ particles. They can be described as open-string states. In a similar manner, collective excitations of the color-singlet sector can give rise to QED mesons. The energies of the lowest QED meson states have been estimated. There are encouraging pieces of evidence for the occurrence of QED mesons as the anomalous X17 and E38 particles observed recently. On-going experiments to confirm these QED mesons are continuing.

The stability of the QED meson states leads to the study of the related QED neutron consisting of two $d$ quarks and a $u$ quark in QED interactions. The attractive QED interaction between the $u$ quark and the two $d$ quarks overwhelms the repulsion between the two $d$ quarks to stabilize the QED neutron at an estimated mass of $44.5 \mathrm{MeV}$. The analogous QED proton has been found to be unstable, and it does not provide a bound state for the QED neutron to decay onto by way of the weak interaction. Hence the QED neutron may be stable against the weak interaction. It may have a very long lifetime and may be a good candidate for the dark matter. Because QED mesons and QED neutrons may arise from the coalescence of deconfined quarks during the deconfinement-to-confinement phrase transition in different environments such as in high-energy heavy-ion collisions, neutron-star mergers [17], and neutron star cores [18], the search of the QED bound states in various environments will be of great interest.

\section{References}

[1] J.Schwinger, Gauge invariance and mass II, Phys. Rev. 128, 2425 (1962).

[2] J.Schwinger, Gauge theory of vector particles, Trieste Lectures, 1962 (IAEA, Vienna, 1963) p. 89.

[3] V.N.Gribov, Local confinement of charge in massless QED, Nucl. Phys. 206, 103 (1982).

[4] V.N.Gribov, The theory of quark confinement,Eur.Phys. Jour.C10,91(1999),[arxiv:hep-ph/9807224]

[5] K.G.Wilson, Confinement of quarks, Phys. Rev. D19, 2445 (1974).

[6] G.Magnifico et al.,Lattice QED in (3+1)dimensions at finite density with tensor networks, [arXiv:2011.10658].

[7] C.Y.Wong,Introduction to high/-energy heavy/-ion collisions, (World Scientific,Singapore,1994).

[8] C.Y. Wong,Anomalous soft photons in hadron production, Phys.Rev.C81,064903(2010),[arXiv:1001.1691].

[9] C.Y.Wong, Open string QED meson description of X17 and dark matter,JHEP08(2020)165,[arxiv:2001.04864].

[10] PDG2019, M. Tanabashi et al., Review of Particle Physics, Phys. Rev. D98, 030001 (2019).

[11] A.J.Krasznahorkay et al., Observation of anomalous internal pair creation in ${ }^{8} \mathrm{Be}$ : a possible indication of a light, neutral boson, Phys. Rev. Lett. 116, 042501 (2016), [arXiv:1504.01527].

[12] K.Abraamyan et al., Check of the structure in photon pairs spectra at the invariant mass of about 38 $\mathrm{MeV} / \mathrm{c}^{2}$, EPJ Web of Conf. 204, 08004 (2019).

[13] DELPHI Collaboration, Direct soft photon production on the jet characteristics in hadronic $Z^{0}$ decays, Eur. Phys. J. C67, 343 (2010), [arXiv:1004.1587].

[14] C.Y.Wong, On the stability of the open-string $Q E D$ neutron and dark matter, [arxiv:2010.13948].

[15] H.W.Crater and P.VanAlstine,Two body Dirac Equations, Ann. Phys. 148, 57 (1983).

[16] H.Sazdjian,Two-body relativistic quantum mechanics and Bethe-Salpeter equation,J.Math.Phys.28,2618(1987).

[17] A. Bauswein et al., Identifying a first-order phase transition in neutron-star mergers through gravitational waves, Phys. Rev. Lett.122, 061102 (2019).

[18] E. Annala et al., Evidence for quark-matter cores in massive neutron stars, Nat. Phys. 16, 907 (2020) 